\title{
Concentration gradients of monoamine metabolites in human cerebrospinal fluid
}

\author{
ROLF SJÖSTRÖM ${ }^{1}$, JAN EKSTEDT ${ }^{2}$, AND ERIK ÄNGGÅRD \\ From the Psychiatric Research Center, Ulleråker Hospital, Department of Neurology, \\ the University Hospital, Uppsala, and Department of Pharmacology, \\ Karolinska Institutet, Stockholm, Sweden
}

SYNOPSIS The monoamine metabolites 5-hydroxyindoleacetic acid (5-HIAA), homovanillic acid (HVA), vanillylmandelic acid (VMA), and 4-hydroxy-3-methoxyphenylglycol (HMPG) were analysed in CSF from different regions of the CSF system to study the caudocranial concentration gradient of the metabolites. Four consecutive $10 \mathrm{ml}$ fractions of CSF were withdrawn in 17 patients during the course of four minutes. The CSF pressure was monitored through a lumbar cannula because of suspected adult hydrocephalus. A pronounced gradient of the HVA concentration was found with a ratio between the last and the first fraction of 1,7. 5-HIAA showed a slight increase while HMPG and VMA showed no increase at higher levels of the CSF system. The results suggest that lumbar HVA reflects dopaminergic activity in the brain, whereas lumbar 5-HIAA and HMPG/ VMA reflect the activity of 5-hydroxytryptamine and noradrenaline secreting neurones in both the brain and the spinal cord.

Estimates of the activity in central monoaminergic neurones obtained by analysis of metabolites of transmitter substances in the cerebrospinal fluid (CSF) have been found useful for the study of several neuropsychiatric diseases -for example, Parkinsonism, Huntington's chorea, and manic-depressive psychosis (Johansson and Roos, 1967; Aquilonius and Sjöström, 1971; Sjöström and Roos, 1972). In these investigations, CSF was usually obtained by lumbar puncture. There is a question, however, whether the lumbar concentrations of the monoamine metabolites reflect the neuronal activity in the brain, the spinal cord or both (Bulat and Zivković, 1971; Sourkes, 1973; Weir et al., 1973).

Guldberg et al. (1966) reported a pronounced gradient of the concentration of 5-hydroxyindoleacetic acid (5-HIAA) - the main metabolite of serotonin (5-HT) - and of homovanillic acid (HVA)-the main metabolite of dopamine

\footnotetext{
1 Address for correspondence: Dr Rolf Sjöström, Psychiatric Research Center, Ulleråker Hospital, S-750 17 Uppsala, Sweden.

2 Present address: Department of Neurology, the University Hospital, Umeå, Sweden.

(Accepted 10 February 1975.)
}

(DA) - from the cisterna magna to the laterat or ventricle in the dog. For 5-HIAA the ratio of ic ventricular to cisternal concentration was $7: 1$, while that for HVA was 29:1. Later Moir et al. (1970) reported a similar gradient from lumbar to ventricular CSF in man, indicating a ventricular/lumbar ratio of about 9:1 for HVA and 5:1 for 5-HIAA. The ventricular CSF was obtained from patients undergoing ventricular catheterization and was compared with lumbar CSF from other patients.

Further studies on the nature of this proposed caudocranial gradient of the concentrations of the amine metabolites seemed desirable. An opportunity for such a study is offered in patients with a suspected adult hydrocephalus where relatively large quantities of CSF are withdrawn for the purpose of lowering the intracranial pressure.

In the present investigation CSF from different levels in the spinocisternal system was analysed for its concentration of 5-HIAA, HVA, and two metabolites of noradrenaline (NA), 4-hydroxy3-methoxyphenylglycol (HMPG) and vanillylmandelic acid (VMA). 


\section{METHODS}

Seventeen patients in a neurological ward participated in the investigation (11 male, seven female; age: 29-72 years). Their clinical state raised the possibility of an adult hydrocephalus. In 10 of the patients the CSF pressure was found to be above the normal level of $1.6 \mathrm{kPa}$. The pressure was recorded through a lumbar cannula with the patient in a supine position for a minimum of $\mathbf{3 0}$ minutes. Afterwards the patient was raised to a sitting position and $40 \mathrm{ml}$ CSF were withdrawn in $10 \mathrm{ml}$ portions (fraction $\mathrm{I}=0-10, \mathrm{II}=10-20, \mathrm{III}=20-30, \mathrm{IV}=30-40 \mathrm{ml}$ ) within four minutes.

5-HIAA was analysed according to Sharman (1960) from nine patients. HVA and HMPG were determined from eight patients with mass fragmentographic techniques (Sjöquist et al., 1973, 1975) and VMA in four of these (Sjöquist, 1975). One patient was investigated twice. The HMPG values are given as the total concentration of HMPG (free and conjugated).

The HVA and HMPG results were treated statistically with paired $t$ tests on the intraindividual differences between the first and each of the subsequent fractions. Because of a pronounced skewness in the distribution of the 5-HIAA differences a nonparametric test was used (Wilcoxon matched pairs rank test; Siegel, 1956).

\section{RESULTS}

The results in the Table and the Figure show an apparently linear increase of HVA from fraction I to IV. The mean ratio between fraction IV and fraction I was 1,7 (range 1,2-2,4). The increase in 5-HIAA was less pronounced (mean ratio fraction IV to I: 1,2). The HMPG concentration did not increase. A statistical analysis was

TABLE

CONCENTRATIONS OF 5-HIAA, HVA, HMPG, AND VMA IN CSF IN FRACTIONS I-IV (MEANS \pm SEM)

\begin{tabular}{lccccc}
\hline Metabolite & $\begin{array}{c}\text { Investiga- } \\
\text { tions } \\
\text { (no.) }\end{array}$ & $I$ & $I I$ & $I I I$ & $I V$ \\
\cline { 3 - 6 } & 9 & $175 \pm 15$ & $188 \pm 15$ & $203 \pm 20$ & $207 \pm 20 \ddagger$ \\
5-HIAA & 8 & $255 \pm 47$ & $348 \pm 51 \ddagger$ & $391 \pm 50 \dagger$ & $440 \pm 46^{*}$ \\
HVA & 8 & $66 \pm 10$ & $68 \pm 10$ & $68 \pm 8$ & $71 \pm 11$ \\
HMPG & 4 & $6 \pm 0.9$ & $4 \pm 0.3$ & $5 \pm 0.6$ & $7 \pm 2.8$ \\
VMA & & & & & \\
\hline
\end{tabular}

$* \mathrm{P}<0.001$.

$+\mathrm{P}<0.01$.

$\ddagger P<0.05$ compared with fraction $I$.

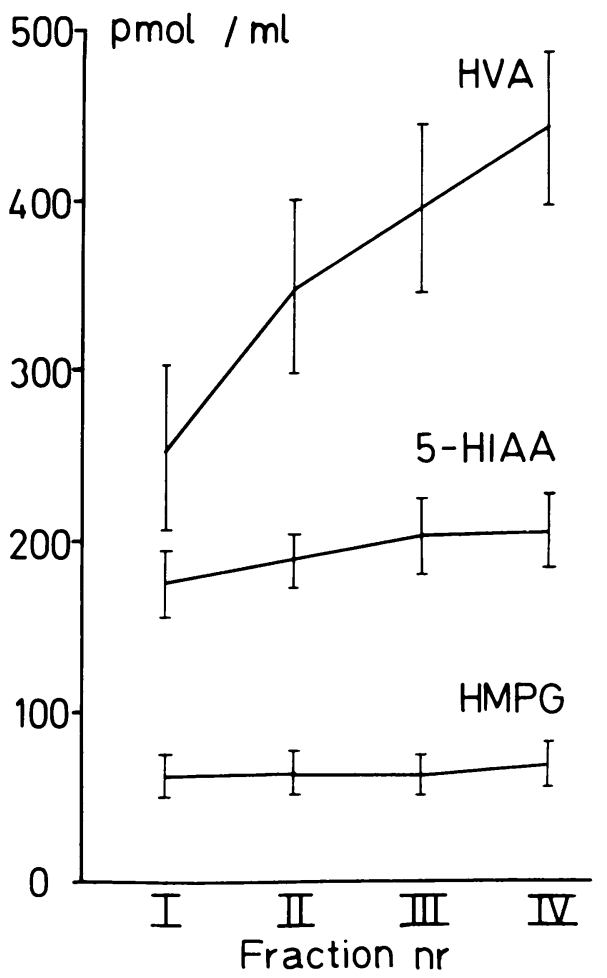

FIGURE Concentrations of $H V A$, 5-HIAA, and $H M P G$ in different regions of the CSF system. Fraction $I=0-10, I I=10-20, I I I=20-30, I V=30-40$ ml CSF. Means \pm SEM.

not made on the VMA data because of the low number of observations. However, the data did not indicate a caudocranial gradient in the concentration of VMA. The VMA concentration was about $8 \%$ of that of HMPG. There was no correlation between the intracranial pressure and the ratio: fraction IV/I.

\section{DISCUSSION}

The total volume of CSF in humans has been estimated at $140 \mathrm{ml}$ (Last and Tompsett, 1953) with about $30 \mathrm{ml}$ in the spinal subarachnoid space (Weston, 1916). The ventricular volume has been estimated at $22 \mathrm{ml}$ (range 7-57) (Last and Tompsett, 1953). This indicates that in the present investigation the first three fractions of CSF were taken from the spinal subarachnoid space, while the last fraction was of cisternal 
origin or from the subarachnoid space surrounding the brain, probably with minimal contribution from the ventricles.

There are virtually no dopaminergic (DA) neurones in the spinal cord (Fuxe et al., 1969). Therefore the HVA in the low region (fraction I) probably originates from the brain, mainly the basal ganglia. The caudocranial gradient of the HVA concentration shown in this investigation confirms earlier reports. It is probably established through bulk flow and active transport of the acid out of CSF during the flow from the ventricles to the lumbar region. Therefore determinations of lumbar HVA concentrations give an attenuated but representative impression of dopaminergic activity in the brain and drug induced changes in the activity in the brain DA neurones should be detectable in lumbar CSF, provided that the method for determination of HVA has satisfactory sensitivity and precision. Changes in HVA concentration may be amplified by the probenecid technique (Sjöström and Roos, 1972). Probenecid inhibits the active transport of HVA out of CSF (Guldberg et al., 1966) and thus increases the concentration in lumbar CSF and decreases the ratio of ventricular to cisternal HVA (Guldberg et al., 1966).

In contrast with HVA, only a small gradient was observed in 5-HIAA and none in HMPGVMA levels at higher regions in the CSF system. This is in contrast with earlier reports of a steep gradient of 5-HIAA between lumbar and ventricular CSF (Guldberg et al., 1966; Moir et al., 1970). A partial explanation of this difference is that ventricular CSF was probably not included in our fraction. Our results demonstrate the likelihood of a relatively pronounced contribution of 5-HIAA and HMPG-VMA from the spinal cord. This is in line with previous work demonstrating both 5-HT and NA neurones in the spinal cord (Fuxe et al., 1969). The levels of 5-HIAA and HMPG-VMA in lumbar CSF therefore only partially reflect activity within 5-HT and NA neurones in the brain, although the small increase in 5-HIAA in the fraction IV indicates a minor contribution of this compound from the brain.

\section{REFERENCES}

Aquilonius, S.-M., and Sjöström, R. (1971) Cholinergic and dopaminergic mechanisms in Huntington's chorea. Life Sciences, pt 1, 10, 405-414.

Bulat, M., and Zivković, B. (1971). Origin of 5-hydroxyindoleacetic acid in the spinal fluid. Science, 173, 738-740.

Fuxe, K., Hökfeldt, T., and Ungerstedt, U. (1969). Distribution of monoamines in the mammalian central nervous system by histochemical studies. In Metabolism of Amines in the Brain, pp. 10-22. Edited by G. Hooper. Macmillan: London.

Guldberg, H. C., Ashcroft, G. W., and Crawford, T. B. B. (1966). Concentrations of 5-hydroxyindolylacetic acid and homovanillic acid in the cerebrospinal fluid of the dog before and during treatment with probenecid. Life Sciences, 5, 1571-1575.

Johansson, B., and Roos, B.-E. (1967). 5-hydroxyindoleacetic acid and homovanillic acid in the cerebrospinal fluid of healthy volunteers and patients with Parkinson's syndrome. Life Sciences, 6, 1449-1454.

Last, R. J., and Tompsett, D. H. (1953). Casts of the cerebral ventricles. British Journal of Surgery, 40, 525-543.

Moir, A. T. B., Ashcroft, G. W., Crawford, T. B. B., Eccleston, D., and Guldberg, H. C. (1970). Cerebral metabolites in cerebrospinal fluid as a biochemical approach to the brain. Brain, 93, 357-368.

Sharman, D. F. (1960). The Significance of Pharmacologically Active Amines in Human Tissues and Body Fluids. PhD Thesis: University of Edinburgh, Edinburgh.

Siegel, S. (1956). Nonparametric Statistics in the Behavioral Sciences. McGraw-Hill: New York.

Sjöquist, B. (1975). Mass fragmentographic determination of vanillomandelic in human urine, cerebrospinal fluid and serum using a deuterium labelled internal standard. Journal of Neurochemistry, 24, 199-201.

Sjöquist, B., Lindström, B., and Ånggård, E. (1973). Mass fragmentographic determination of homovanillic acid in tissues and body fluids using the deuterium labeled species as internal standard. Life Sciences, 13, 1655-1664.

Sjöquist, B., Lindström, B., and Ȧnggård, E. (1975). Mass fragmentographic determination of 4-hydroxy-3-methoxyphenylethylene glycol in tissues and body fluids. Journal of Chromatography, 105, 309-316.

Sjöström, R., and Roos, B.-E. (1972). 5-hydroxyindolacetic acid and homovanillic acid in cerebrospinal fluid in manic-depressive psychosis. European Journal of Clinical Pharmacology, 4, 170-176.

Sourkes, T. L. (1973). On the origin of homovanillic acid (HVA) in the cerebrospinal fluid. Journal of Neural Transmission, 34, 153-157.

Weir, R. L., Chase, T. N., Ng, L. K. Y., and Kopin, I. J. (1973). 5-hydroxyindoleacetic acid in spinal fluid: relative contribution from brain and spinal cord. Brain Research, 52, 409-412.

Weston, P. G. (1916). Sugar content of the blood and spinal fluid of insane subjects. Journal of Medical Research, 35, 199-208. 